\title{
RECENT REPORT ON THE ASCA GIS SOURCE CATALOG PROJECT
}

Y. ISHISAKI AND T. OHASHI

Department of Physics, Tokyo Metropolitan University

1-1 Minami-Osawa, Hachioji, Tokyo 192-03, Japan

Y. UEDA AND T. TAKAHASHI

The Institute of Space and Astronautical Science

3-1-1 Yoshinodai, Sagamihara, Kanagawa 229, Japan

K. MAKISHIMA

Department of Physics, University of Tokyo

7-3-1 Hongo, Bunkyo-ku, Tokyo 113, Japan

AND

\section{THE GIS TEAM}

\section{Introduction}

We are constructing the ASCA GIS source catalog from the ASCA public archive, mainly for extra-galactic sky. The large field of view and the lowbackground characteristics of the GIS make it suitable for a search for serendipitous sources in a wide energy band of $0.7-10 \mathrm{keV}$. Sources to be detected by the project will provide valuable information on the $\log N-\log S$ relation over the entire sensitivity band, which has never been available before. About this project, also refer to Ishisaki et al. (1995), Ueda et al. (1997) and Takahashi et al. (1997). There is the SIS source catalog project, too (Gotthelf et al., 1996). These catalogs are going to appear on the WEB.

\section{Field Selection and Present Progress}

The automatic source-finding procedure has been applied to all the archival data (1993-1996) which satisfy the following selection criteria; the Galactic latitude $|b|$ is higher than 10 degrees, the net exposure is longer than $10 \mathrm{ks}$, and the primary target is less bright than $10 \mathrm{c} / \mathrm{s} /$ GIS in the total count rate. Data out of 481 pointings which amount to $262 \mathrm{deg}^{2}$ have been analyzed so far. After running the automatic process, we further rejected the field where the results of the 2-dimensional fitting turned out to be unacceptable. With 
these filters, we detected 992 sources above $5 \sigma$ detection in $0.7-7$ and/or 2-10 $\mathrm{keV}$ band including the main targets.

\section{Derived $\log N-\log S$ Relation}

The detected source fluxes distribute in a wide range from $10^{-14}-10^{-10} \mathrm{erg}$ $\mathrm{s}^{-1} \mathrm{~cm}^{-2}(2-10 \mathrm{keV})$. To convert flux distribution to the $\log N-\log S$ relation, we must carefully estimate the survey area at this detection limit, as well as the influence of the main target. To reduce the influence of the main targets, we further selected 27 fields observed in 1994 with severer conditions that $|b|>50^{\circ}$ and the primary target is less bright than $0.25 \mathrm{c} / \mathrm{s} /$ GIS. To estimate the detection significance for a given flux, we utilized a simplified simulation at every position $(4 \times 4$ arcmin cell in practice). Figure 1 shows the derived $\log N-\log S$ relation from the $2-10 \mathrm{keV}$ band survey of the selected 27 sample fields. Although the contributions of the main targets are not excluded, our result lies on the extrapolation from the results of Ginga fluctuation analysis (Stewart 1992) and the source counts by HEAO1 A2 (Piccinotti et al. 1982), which is consistent with the ASCA Large Sky Survey (LSS) results (Ueda 1996).

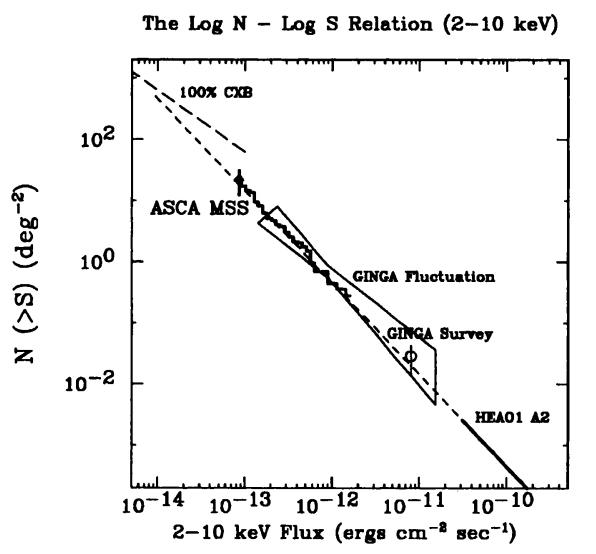

Figure 1. The 2-10 keV $\log N$ - $\log S$ relation (labeled "ASCA MSS") derived from the selected 27 sample fields. Main targets are also included. $4 \sigma$ significance detection level are chosen for this plot.

\section{References}

Gotthelf, E. and White, N. G. (1996), in "X-ray Imaging and Spectroscopy of Cosmic Hot Plasmas" ed F. Makino and K. Mitsuda (Universal Academy Press, Tokyo)

Ishisaki, Y., Takahashi, T., Kubo, H., Ueda, Y., Makishima, K., and the GIS team (1995), ASCA News No. 3, 19.

Piccinotti, G. et al. (1982), Astrophys. J., 269, 423.

Stewart, G.C. 1992, in The X-Ray Background, eds. X. Barcons and A.C. Fabian (Cambridge University Press: Cambridge), p187.

Takahashi, T., Ueda, Y., Ishisaki, Y., Makishima, K., Ohashi, T. (1997), in "X-Ray Surveys" workshop at Potsdam, Germany

Ueda, Y. (1996), PhD. thesis, The University of Tokyo

Ueda, Y., Takahashi, Y., Ishisaki, Y., Makishima, K., Ohashi, T. (1997), in "All-Sky X-Ray Observations in the Next Decade" workshop at RIKEN Saitama Japan 